\title{
A Road Map to Understand the Current Status of Diabetic Retinopathy Detection and Classification Methods

\author{
Nitin M Shivsharan, Sanjay Ganorkar
}

\begin{abstract}
In the working-age population, the primary reason for blindness is Diabetic Retinopathy, which can be identified from the retinal fundus image. So far, more research works had been progressed for $D R$ early detection since it is vital to save the vision of diabetes patients. This research work has made a short survey on diverse DR detection mechanisms for determining the shortcoming of contributed papers to promote the future scope of this research aspect. The overall analysis is made with respect to chronological review, performance parameter analysis, and algorithmic analysis used in different contributed papers. The obtained best performance values are also clearly determined. Moreover, the research gaps and challenges associated with this diabetic retinopathy detection and classification methods are discussed further, which directly paves way for future progressions.
\end{abstract}

Keywords: Diabetic Retinopathy; Microaneurysms; DR Detection and Classification; Algorithmic Analysis; Challenges

\begin{tabular}{|c|c|}
\hline \multicolumn{2}{|r|}{ Nomenclature } \\
\hline Abbreviations & Descriptions \\
\hline CNN & Convolutional Neural Network \\
\hline DR & Diabetic Retinopathy \\
\hline PNN & Probabilistic neural network \\
\hline ECOC & Error-Correcting Output Codes \\
\hline T-LOP & Tetragonal Local Octa Pattern \\
\hline ELM & Extreme Learning Machine \\
\hline ReLU & Rectified Linear Activation Unit \\
\hline ROC & Receiver Operating Characteristic \\
\hline $\mathrm{ACO}$ & Ant Colony Optimization \\
\hline VG & Visibility Graph \\
\hline RDR & Referable Diabetic Retinopathy \\
\hline BoVW & Bag-of-Visual-Words \\
\hline MIL & Multiple Instance Learning \\
\hline RT & Radon Transform \\
\hline GA & Genetic algorithm \\
\hline SOM & Self-Organizing Map \\
\hline AHE & Adaptive histogram equalization \\
\hline HSOM & Hierarchical SOM \\
\hline KNN & K Nearest Neighbor \\
\hline RF & Random Forest \\
\hline BEDC & Bi-Elliptical Deformable Contour \\
\hline
\end{tabular}

Revised Manuscript Received on March 16, 2020.

* Correspondence Author

Nitin M Shivsharan*, E \& TC Dept Sinhgad College of Engineering, Pune, India. shivsharan.nitin@gmail.com

Sanjay Ganorkar, E \& TC Dept Sinhgad College of Engineering, Pune, India. srganorkar.scoe@sinhgad.edu

(C) The Authors. Published by Blue Eyes Intelligence Engineering and Sciences Publication (BEIESP). This is an open access article under the CC BY-NC-ND license (http://creativecommons.org/licenses/by-nc$\underline{\mathrm{nd} / 4.0 /)}$

\begin{tabular}{|l|l|}
\hline FCM & Fuzzy C Mean \\
\hline GSO & Glowworm Swarm Optimization \\
\hline ML-BEC & Machine Learning Bagging Ensemble Classifier \\
\hline SVM & Support Vector Machine \\
\hline t-SNE & t-distributed Stochastic Neighbor Embedding \\
\hline RI & Retinal Image \\
\hline OD & Optical Disk \\
\hline MA & Microaneurysm \\
\hline PDR & Proliferative DR \\
\hline NPDR & Non-Proliferative DR \\
\hline NPDR & Non-proliferative Diabetic Retinopathy \\
\hline GMM & Gaussian Mixture Model \\
\hline CWS & Cotton Woolspots \\
\hline CHT & Circular Hough Transform \\
\hline HE & Hard Exudates \\
\hline DL & Deep Learning \\
\hline ANN & Artificial neural network \\
\hline AUC & Area Under Curve \\
\hline
\end{tabular}

\section{INTRODUCTION}

Patients having high diabetes history are more prompt to DR. Owing to DR, the patients tends to have high level sugar in the blood, by which the blood circulation through the tiny blood vessels across the retina is jammed. These injured vessels affect the retinal region with a shortage of blood that can direct to everlasting vision loss. Early signs of warning in account of DR are not available until it affects the vision. The broad division of DR is of two phases, i.e. NPDR and PDR. NPDR, also named as background DR, arises while diabetes impacts the blood vessels within the retina, which often leads to leakage of fluid and blood on the retinal surface. The retinal function minimizes as it gets swollen and wet because of this leakage. NPDR can comprise of diverse retinopathy signs like hemorrhages, MAs, soft exudates or CWS and HE.

The prime indication of NPDR is MAs that impacted by thin vessel dilations. The appearance of MAs is in the shape of round with red color, and smaller size. The subsequent DR indication is HMs named as blot or dot HMs. While the damage of slender vessels walls or MAs is severe, the HMs ratio increases that leads them to explode. HMs is appeared to be in small clear red dots while comparing over blot HMs that appeared in higher red lesions. Sometimes dot HMs and MAs appeared as a red single lesion called HMAs. The occurrence of DR is made in two diverse stages. NPDR is the initial stage that is very dangerous to retina because of the vessel perforation causes them for excreting a substance owing to over glucose accumulation.

Published By:

Blue Eyes Intelligence Engineering \& Sciences Publication

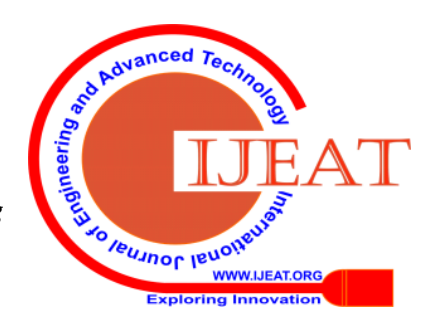




\section{A Road Map to Understand the Current Status of Diabetic Retinopathy Detection and Classification}

Methods

The retina is made damped by leaked fluid, which results in regular loss of eyesight. The severity of damage is depicted on the basis of NPDR stages.

In the view of quantity and presence of these lesions, the further classification of NPDR was exploited under three phases called moderate, mild, and severe. The detection in early stage and its corresponding treatment is still seems very crucial as it is considered as a progressive disease. Furthermore, as per the availability of count and kinds of lesions within the fundus image, the severity may get identified. In the other hand, a healthy retina is comprised of optic disc, blood vessels, and macula as its core components and if any variations in these components lead to the signs of any eye disease.

The contribution is summarized as follows:

- This survey intends to make a review on DR detection and classification for determining the limitations of contributed papers.

- Various analyses are made regarding the performance used in the contributed papers.

- $\quad$ Further, the best performance value among the parameters is also listed in this work.

- Further, the research gaps and challenges regarding the DR detection and mechanism are explained for future advancement.

This survey arrangement is listed as below: Section II depicts the literature review on the paper that contributed to this survey. Section III explains the short review of adopted algorithms, performance measure and maximum attainment. Section IV discusses research gaps and challenges. Section $\mathrm{V}$ concludes this research work.

\section{LITERATURE SURVEY}

\subsection{Related Works}

In 2018, Wan et al. [1] have intended on determining an automated classification method for a provided set of fundus images. In this, DR detection was proliferated via the CNNs that contain three main critical challenges like segmentation, classification, and detection. This method has adopted VggNet, ResNet, GoogleNet, AlexNet, and analyzed these models in coupling with hyper-parameter and tuning transfer learning for determining the DR image classification. Further, Kaggle platform is deployed to train these methods. The classification accuracy was attained to the beast as $95.68 \%$ and the outcomes have represented the superior transfer learning and CNN accuracy on image classification of DR.

In 2017 Amin et al. [2] has introduced a structure that automatically classifies the nonexudates and exudates regions within retinal images and was on the basis of feature extraction, classification and pre-processing. Gabor filter was adopted in pre-processing for grayscale image that enhances the lesion. On the basis of mathematical morphology, the candidate lesion segmentation was performed. The selection of features set was made under every candidate lesion based on the geometric and statistical features. The performance of the introduced model was evaluated under false positive, true positive and statistical analysis curve and thus explained with better accuracy.

In 2013, Akram et al. [3] have implemented a threestage system based on filter banks for MA's early detection. chronological review and parameter

Initially, the MA's entire probable candidate regions were extracted by the system within retinal image. A feature vector for every region was formulated for classifying the candidate region as non- MA or MA based on specific features like color, statistics, intensity, and shape. A hybrid classifier model has been proposed that incorporates the SVM, GMM, and expansion of multimodel mediod depended modeling technique in an ensemble for improving the classification accuracy. The evaluation of implemented system was made and attained with larger accuracy than other published models.

In 2014, Akram et al. [4] have introduced a new hybrid classifier model for effective retinal lesion detection. This model involved feature set formulation, preprocessing, classification and extraction of candidate lesions. The filter banks were deployed for extracting the entire regions having lesions in the candidate lesion detection phase. For better classification, a feature set on the basis of diverse descriptors like intensity, shape, and statistics, was exploited for every possible candidate region. In this research work, an expansion of m-Mediods based modeling technique was presented for forming a hybrid classifier by merging with Gaussian Mixture method for enhancing the classification accuracy.

In 2015, Welikala et al. [5] has introduced an automated model for detecting the novel vessels from retinal images and was depending on dual classification technique. Two separate binary vessel map that embraced core information was created by applying the two vessel segmentation techniques. The features of Local morphology had been estimated for producing two individual 4-D feature vectors from every binary vessel map. For every feature vector, independent classification was carried out by means of an SVM. The final decision was offered by combining these individual results. Finally, the performance under this model has revealed the better sensitivity and specificity results on a per-image basis.

In 2019, Nazir et al. [6] have established a narrative approach for the precise detection of diverse DR stages by expanding the image retrieval research based on content domain. The fundus images were demonstrated by the new T-LOP features for attaining the performance at humanlevel under huge-scale DR-datasets, which were then classified via the ELM. The established model was differentiated over various conventional models like deep learning models under four DR-datasets having variational lengths for justifying the consequence of the model. The simulation outcomes have ensured the DR-detection schemes significance for serving as a stand-alone solution to provide accurate information on DR severity in an effective manner.

In 2019, Shanthi and Sabeenian [7] has concerned about the DR fundus image classification in accordance with the disease based on CNN having the application of Softmax, suitable Pooling, and ReLU layers for attaining a large accuracy level. Messidor database was deployed for validating the performance of the adopted algorithm. From the results, owing to healthy images, the classification accuracy of $96.6 \%$ and $96.2 \%, 95.6 \%$ and $96.6 \%$ have been obtained by the images of phase 1 , phase 2 and phase 3 of DR. 
In 2019, Pires et al. [8] have examined the data-driven approaches which derive the representation of powerful abstract straight from retinal images for providing a dependable referable detector on diabetic retinopathy. On the basis of $\mathrm{CNN}$, the solution has been build gradually by adding vigorous feature-extraction augmentation, multiresolution training, data augmentation, a patient-basis analysis, and validating the efficiency of every enhancement. The implemented approach has attained an area within a stern cross-dataset protocol under the ROC curve of $98.2 \%$. Same outcomes were attained for DR2 and Messidor-2 datasets having $5 \times 2$-fold cross-validation protocol that in turn reduced the classification error as $44 \%$ on comparing over other conventional models.

In 2019, Selcuk and Alkan [9] have aimed on detecting microaneurysms effectively, accurately and automatically in early stage that was critical for detecting in color fundus images. Rather than the existing image processing approaches, this research work used the ACO approach, which was considered as an effective meta-heuristic algorithm. Initially, from color fundus images, the extraction of retinal vascular structure was made using DiaretDB1 and Messidor data sets. Then, the ACO algorithm was deployed for the effective segmentation of microaneurysms. The other five diverse clustering and image processing algorithms were applied with the same process and then compared over the proposed model. The proposed ACO algorithm has validated a more stabilized and larger image contrast performance irrespective. Thus, the implemented model evidently identifies the microaneurysms still in low-quality images.

In 2019, Mohammadpoory et al. [10] have proposed a model on the basis of VG and RT for automatically discriminating to grades 0 (normal), 1,2 and 3 of the fundus image DR. The summarization of implemented model was made in two phases like classification and feature extraction. For the first time, the VG model was employed in this study for feature extraction in the field of image processing. Subsequently for classification purpose, these features have been provided over ECOC model. The implemented model was achieved with a specificity, sensitivity and accuracy of 98.61\%, 95.83\% and 97.92\%.

In 2016, Seoud et al. [11] have described and validated the model for the detection of hemorrhages and microaneurysms automatically in color fundus images. The core objective was in the introduction of novel shape features' set named Dynamic Shape Features, which has not needed accurate region segmentation were classified. The shape evolution was represented by these features at the time of image flooding and permitted for discriminating among vessel segments and lesions. The validation of this method was made per image and per-lesion by means of six databases and thus validated the robustness regarding quality, variability in image resolution, and acquisition system.

In 2014, Zhang et al. [12] have established a noninvasive model for predicting DM and NPDR, which was the prime DR phase on the basis of three clusters of features derived from tongue images, which was comprised of texture, color, and geometry. By deploying a non-invasive capture device with image correction, the capturing of tongue images was initially made. An establishment of color gamut of the tongue was made using twelve colors demonstrating the color characteristics of tongue. The simulation experiment has evaluated that the implemented method has separated the NPDR/DM-sans NPDR tongues and also Healthy/DM tongues based on the features from every three groups and that has attained average accuracy of $80.33 \%$ and $80.52 \%$, correspondingly.

In 2019, Zeng et al. [13] have evolved a computer-aided diagnosis model for deep learning algorithms for diagnosing automatically the RDR by categorizing the color photographs of retinal fundus within two grades. This research work has trained the Siamese like architecture using a narrative CNN method having transfer learning approaches. This adopted method apart from earlier works has accepted the input as binocular fundus images and studied their relationship, in order to aid on prediction making. A binocular model was as well evaluated and trained for the further verification of the binocular design effectiveness for achieving the five-class DR detection. The outcomes thus demonstrated the better attainment of kappa score as 0.829 that was larger than that of conventional nonensemble methods.

In 2013, Pires et al. [14] have presented an algorithm for making the decision on the basis of the result fusion using meta-classification. The meta-classifier input was assigned for the retinal images as the outcome of various lesion detectors, generating a dominant large level feature demonstration. Further, the options for BoVW depended lesion detectors were developed, where crucially on the basis of the low-level local descriptor's options of pooling and coding. The concluded classification technique has attained an AUC of $93.4 \%$ by means of SOFTMAX BoVW (max pooling /soft-assignment coding), with no requirement of normalization of large-level feature score vectors.

In 2012, Antal and Hajdu [15] have formulated an ensemble-based model for enhancing the detection of microaneurysm. Further, this model introduced an amalgamation of internal modules of microaneurysm detectors called candidate extractors and preprocessing models, apart from the eminent technique of taking the multiple classifier output. The evaluation of this implemented technique was made of the detection of microaneurysm within an online competition. Given that the detection of microaneurysm was crucial in DR grading, the testing of implemented model for this task was he publicly made on existing Messidor database, in which precisely attained the "DR/non-DR"-kind classification depending on the existence or absence of microaneurysms.

In 2018, Costa et al. [16] a novel approach has been introduced on the basis of MIL framework performed at the image level for defeating this requirement by influencing the implied information available on annotations. In contrast over the earlier MIL-based DR detection systems, this method has incorporated the core contribution as the merged optimization of image classification stages and the instance encoding. Using this way, the obtainment of a more helpful mid-level demonstration of pathological images was made. The model decision explainability was improved further using a novel loss function that enforced suitable instance and mid-level demonstrations. The implemented approach has attained superior outcomes over the other recent conventional models, whereby enhances the produced decision interpretability. 


\section{A Road Map to Understand the Current Status of Diabetic Retinopathy Detection and Classification}

Methods

In 2018, Zhou et al. [17] have established a deep MIL model to predict DR, where studied the classifiers and features from data to detect the DR images with their internal lesions and has attained a considerable enhancement. Particularly, a pre-trained CNN was applied for attaining the estimation of patch-level DR, after that global aggregation was utilized for performing the image classification in DR. Moreover, for the enhanced dealing of unequal DR lesions, an end-to-end multi-scale system has been proposed. In order to attain the precise DR image detection, this model has attained an AUC curve 0.960 on Messidor and 0.925 over a Kaggle dataset subset. For the effective DR lesions prediction, the proposed model has achieved as $0.924 \mathrm{~F} 1$-score with sensitivity and precision as 0.995 and 0.863 on DIARETDB1 by means of associated component-level validation.

In 2019, Hemanth et al. [18] have introduced a solution model that consisted of histogram equalization for image processing employment, and the contrast restricted adaptive histogram equalization approaches. After that, based on the classification of a CNN, the diagnosis was carried out. By taking 400 retinal fundus images within the MESSIDOR database, this model has been verified. The parameters with average value performance has been achieved in terms of sensitivity (recall) as $94 \%$, accuracy as $97 \%$, precision as $94 \%$, specificity as $98 \%$, GMean as $95 \%$ and FScore as $94 \%$. Additionally, a common comparison was made over the conventional studies have thus demonstrated the effective and successfulness of developed model.

In 2016, Rahim et al. [19] have proposed a narrative fuzzy based model for the identification of DR and maculopathy automatically in eye fundus images. Initially, the existing models were introduced for the screening of DR, based on maculopathy detection models. The implemented system has comprised of four phases, called: image preprocessing has included four retinal structures localization, image acquisition, classification, and feature extraction. In the implemented system, the fuzzy based model along with diverse feature extraction models and CHT were used as the combination. Further, a new approach has been presented for detecting the maculopathy in the macula region localization.

In 2016, Rahim et al. [20] have established a narrative automatic screening scheme for DR that mainly concerned on earlier detection of retinopathy's visible signs like microaneurysms. At first, the available schemes and applications associated with DR screening having the microaneurysm prediction models were explored. The implemented decision support scheme comprised of screening, classification, and automatic acquisition of DR in color fundus images, thereby assisted in managing and detection of DR. In the implemented detection model, diverse feature extraction models and CHT was deployed along with the fuzzy histogram equalization model. In the preprocessing stage, another model has been adopted for DR eye fundus images and offered enhanced outcomes in the detection of microaneurysms.

In 2012, Akram and Khan [21] have implemented the computer-assisted scheme for the early DR prediction. In this, the algorithm was presented regarding the blood vessel enhancement, retinal image preprocessing, segmentation and optic disk localization and detection. Further, the implemented hybrid fuzzy classifier was deployed for the effective detection of diverse DR lesions. Four diverse publicly available databases were deployed for the testing of developed models. The developed model was then differentiated over the recently established models and the outcomes thus demonstrated the betterment.

In 2019, Chakraborty et al. [22] have implemented the supervised learning-based model by means of ANN for attaining more precise detection results in the case of DR. The input that was given to the ANN-based classifier was the features that extracted from the retina images. The accuracy of this method has been enhanced using the Customized ANN architecture that estimated various traditional ANN entities. The architecture of ANN that deployed was the feed-forward back-propagation NN. The implemented model in terms of accuracy has attained betterment of about 97.13\%. The outcomes thus recommended that the implemented model has been deployed for detecting diabetic retinopathy efficiently.

In 2014, Ganesan et al. [23] has formulated the trace transforms to construct a human visual system that could duplicate the image view path of a human observer. In this, the SVM alongside polynomial, quadratic, radial basis function kernels, and PNN were deployed for classifying the features in this approach. In order to finely tune the classification parameters, GA was employed. From the overall analysis, the result thus showed the implemented model with better accuracy for SVM quadratic kernels and PNN-GA.

In 2018, Pang et al. [24] have implemented a novel approach that enhanced interpretability via visualization on the basis of results, and that enhanced the accessibility via a message queue framework and web services. In addition, owing to this research work, a report system has been modeled for doctors and was modeled as a mobile APP for patients. By this mean model, the proposed method has been validated in actual practices.

In 2019, Zadeh et al. [25] have introduced a classification scheme that comprised the narrative permutation of SOM to detect the retinal lesions. The implemented model has comprised of a quick preprocessing phase chased by feature extraction of lesion and, lastly, a detailed classification method. On considering the pre-processing phase, three processes were existed called optical disk extraction, target lesion extraction, and finally retinal blood vessels extracting from retina. Second, the merging of multiple features like color, morphology, moments and intensity were made. The classifier model that used was HSOM that aided for maximizing the speed and accuracy of lesions classification when assuming the large data quantity in feature extraction. The outcomes have demonstrated that the implemented approach can accurately classify and detect the Lesions in HDR images.

In 2019, Raja and Balaji [26] have developed an effective automatic detection of diabetics by means of CNN in retinal images. The adopted scheme was comprised of five constraints like blood vessel segmentation, preprocessing, texture feature extraction, diabetic detection, and exudates segmentation. AHE was adopted in the preprocessing to enhance the retinal image input. Subsequent to this, CNN and FCM were deployed for the blood vessel segmentation and exudates segmentation, correspondingly. After that,

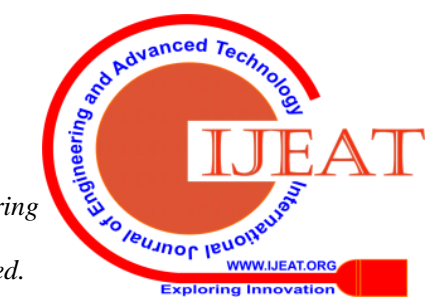


the extraction of texture features was exploited from exudates and blood vessel.

SVM was used for the classification of diabetics. The outcomes thus have shown the developed model has attained superior diabetic detection outcomes than other compared models.

In 2018, Karthikeyan and Alli [27] have introduced an efficient algorithm in image processing for maximizing the efficiency and for identifying the DR diseases. The microaneurysms automatic detection was the main challenge in this task. This work classifies the DR using the SVM parameters optimized with GSO and GA. For controlling the classifier performance, the SVM was combined with hybrid GSO-GA alongside feature chromosomes. The experimental outcomes have thus validated the enhancement of the proposed model with superior performance.

In 2019, Karkuzhali and Manimegali [28] have employed an implemented approach for separating the retina landmark and retina lesions for the stage DR classification. Some of the segmentation methods such as maximum intensity variation, toboggan inverse segmentation, Gabor double-sided hysteresis thresholding, multi-agent approach, and surface adaptive thresholding were utilized for detecting and segmenting EXs, BVs, Has, ODs, and MAs. The machine learning algorithm and feature vector formation were deployed for classifying the several phases of DR utilizing images presented in several retinal databases, and their performance measures.

In 2017, Somasundaram and Alli [29] has designed an early detection and diagnosis tool for DR disease for identifying the retinal features based on Ensemble Classification and Machine Learning model named MLBEC. Two stages were comprised within the ML-BEC approach, where the candidate objects extraction from RI. Then the features were extracted firstly by adopting Machine Learning approaches named, t-SNE. In addition, the images were divided into dissimilar and similar pairs in t-SNE which produces a probability distribution across large-dimensional images. Next, in the low-dimensional map, a similar probability distribution was described by tSNE through the points. Secondly, the application of ensemble classifiers was used for the extraction of features to provide precise analysis of digital FI by means of machine learning. Owing to this phase, a DR screening automatic detection system was exploited by means of BEC was examined.

In 2019, Huang et al. [30] has intended to automatically analyze and extract diverse DF constraints, like the presence of MAs, Euclidian distance among center of the OD and macula, MAs with exudate, retinal condition of eyes and diameter of OD. On assuming the DR attributes, the important DR attributes were firstly determined by adopting fuzzy analytical network procedure for ranking attributes from increased to decreased connected factors to DR. Afterwards, the creation of transformed fuzzy NN was made for enhancing the accuracy on classification. To the end, the association rules between the selected attributes of DR were extracted for revealing their degree and importance of severity. The outcomes of the proposed model have thus shown the superiority of proposed model.

\section{SHORT REVIEW ON ADOPTED ALGORITHMS, PERFORMANCE MEASURES AND MAXIMUM ATTAINMENT}

\subsection{Chronological Review}

Fig. 1 represents the chronological review alongside the contribution percentage regarding the contributed papers on Diabetic retinopathy. The contributed papers are taken and reviewed from the year 2012 to 2019. Maximum number of papers is taken from the year 2019 and that is $40 \%$. From 2018, $16.67 \%$ of the papers related to DR are selected for the review. Only $3.33 \%$ of papers are published in the year 2015. 6.67\% of papers are contributed from the years 2012, 2013, and 2017, similarly, $10 \%$ of the papers are contributed from the years 2014 and 2016.

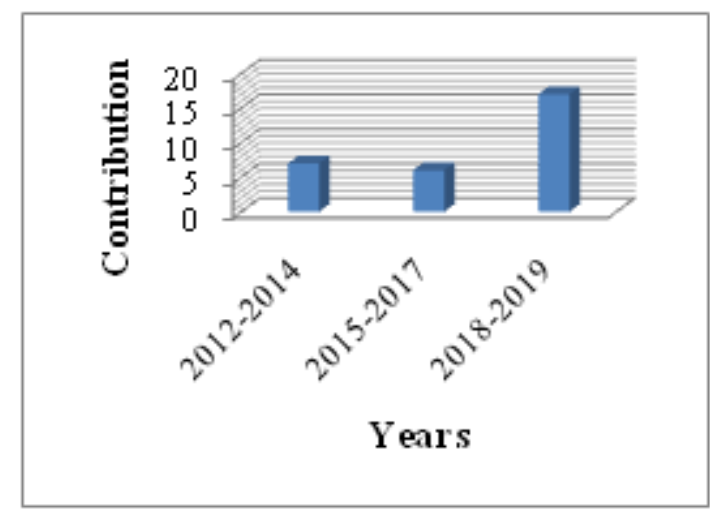

Fig. 1.Chronological review

\subsection{Algorithmic Analysis}

Fig. 2 delineates the survey on various algorithms that are used in the contributed papers. The implemented algorithms are under five categories: Machine Learning algorithms, Optimization algorithms, Hybrid classifier model, Fuzzy model, and others. In machine learning algorithm, some of the contributed models are CNN that are used in [1] [7] [8] [13] [18] [24], KNN is deployed in [2], RF is employed in [11], SVM is utilized in [14] and ANN is used by the authors in [22]. Owing to the optimization algorithms, the author in [5] uses the GA algorithm, whereas ACO algorithm is deployed over the paper [9]. The third one is the hybrid classifier. In this, the author in [3] implements a hybrid classifier that hybrids the concept of GMM and SVM. Similarly, in [4], the GMM along with M-Mediods is combined. In [21], hybrid fuzzy classifier is implemented. In [23], the PNN is combined with the optimization algorithm GA. In [27], SVM classifier is hybrid along with GSO-GA optimization algorithms. Some of the fuzzy models that are deployed in the contributed papers are [19] [20] [26] and [30]. To the end, the contributed papers are reviewed in other categories according to the methodology. T-LOP is the algorithm that is used by the author in [6]. In [10], the ECOC model is implemented. BEDC model is deployed over the paper [12]. In [15], the ensemble-based system is deployed by the author. Improved BoVW is one of the other models inspected in [16]. Deep multiple instance learning is employed in [17]. HSOM map is involved for the detection process in paper [25].

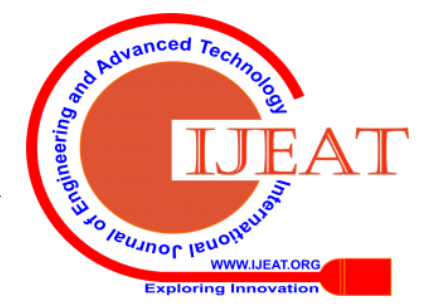


A Road Map to Understand the Current Status of Diabetic Retinopathy Detection and Classification Methods

Gabor double-sided hysteris thresholding is the method deployed in [28] by the author. ML-BEC is the algorithm used for the detection of DR in [29].

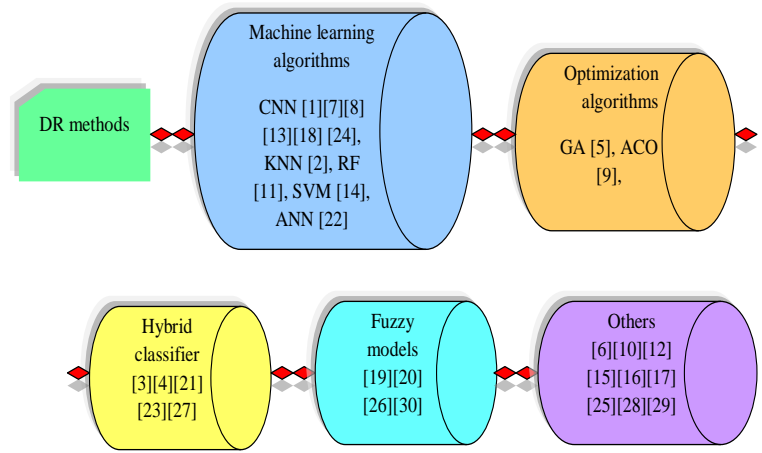

Fig. 2.Analysis on Adopted Algorithms

\subsection{Analysis on Performance Measures}

Table I delineates the analysis on performance measures in the reviewed papers. Some of the core measures that are analyzed in the contributed papers are explained as follows: Accuracy, Sensitivity, Specificity, and AUC. From the overall analysis, the accuracy is the measure that considered in maximum works, which is the $66.67 \%$ of total contribution, $43.33 \%$ of total contribution have used the measures like sensitivity and specificity. The $16.67 \%$ of total contribution has measured AUC, and the other measures are considered in $13.33 \%$ of contribution, which includes precision, recall, computation time, mean, mode, zscore, probability, F-score and so on.

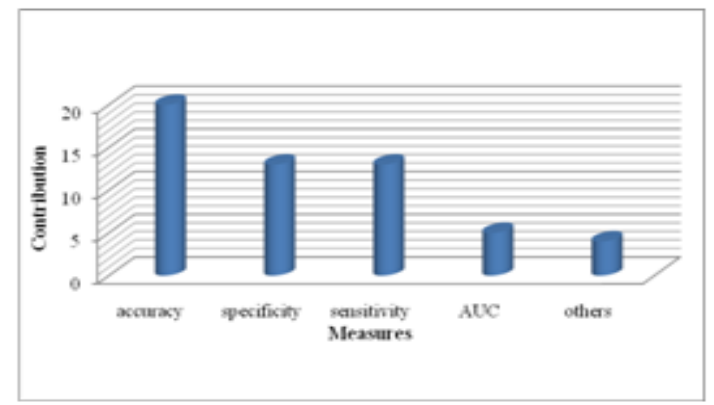

Fig. 3.Analysis on Performance Measures of Reviewed papers

\subsection{Maximum performance}

Table II explains the maximum attained measure by each 0 the reviewed papers. In fact, the basic performance measures in any of the review papers are accuracy, sensitivity, and specificity, respectively. Here, the maximum attained value of accuracy is $98.84 \%$. Similarly, the attained highest value of sensitivity is $95.83 \%$, and the attained highest specificity value is $98.61 \%$. Similarly, the other measures with their best performance value are listed in the Table II.

TABLE I. Obtained maximum perormance

\begin{tabular}{|c|c|c|}
\hline Measure & $\begin{array}{l}\text { Best } \\
\text { performance } \\
\text { value }\end{array}$ & Citation \\
\hline Accuracy & $98.84 \%$ & $\begin{array}{l}\text { [1] [2] [3] [4] [5] [6] [7] } \\
{[10][12][18][19][20]} \\
{[21][22][23]}\end{array}$ \\
\hline Sensitivity & $95.83 \%$ & $\begin{array}{l}{[1][5][7][10][13][18]} \\
{[19][20][25][26][27]}\end{array}$ \\
\hline
\end{tabular}

\begin{tabular}{|l|l|l|}
\hline & & {$[28][29]$} \\
\hline Specificity & $98.61 \%$ & $\begin{array}{l}{[1][5][7][10][17][18]} \\
{[19][20][25][26][27]} \\
{[28][29]}\end{array}$ \\
\hline AUC & $96 \%$ & {$[8][13][15][16][30]$} \\
\hline Precision & 0.863 & {$[6][7][17][18]$} \\
\hline Recall & 0.9883 & {$[6]$} \\
\hline F1-score & 0.9812 & {$[17][18][27]$} \\
\hline z-score & $91.7 \% \pm 2.2 \%$ & {$[14]$} \\
\hline $\begin{array}{l}\text { Computation } \\
\text { time }\end{array}$ & $60 \mathrm{~s}$ & {$[8][11][28]$} \\
\hline Mean & 0.84 & {$[9]$} \\
\hline Probability & 0.9883 & {$[24]$} \\
\hline
\end{tabular}

\section{RESEARCH GAPS AND CHALLENGES}

Amid the working-age adults, DR is considered as a primary reason of blindness. Hence, the DR's early detection is crucial for a better prediction. Some of the future problems are stated as follows: one of the core issues in processing the retinal based DR disease is on the extraction of features. Moreover, the processing time is not considered in most of the works. Hence as future work, more hybrid approaches are in emerging for decreasing the computation time. Another challenge on DR detection is in the implementation of deeper collaborative associations with clinics and hospitals for accessing more relevant data on lesions 3 and 4 . The classification accuracy may attain precise with more data. But from the research, the differentiation among the images lesions 0 and 1 is seemed to be hard. Hence, the collection of more images that belongs to lesions 0 and 1 is necessary while selecting the new data.

Most of the recent image and video processing applications are based on the deep learning algorithms, because of its proven importance. However, the powerful DL network is necessary because of some possible directions that need to be addressed. In reality, DL-based models are as well deployed for the automatic improvement of DR detection system. Various DL models exist in the literature, which chiefly deployed the CNN methods for designing deep multi-layer architectures, for predicting the DR from retinal fundus images. Even though, these image annotation needs ophthalmologists that is much time consuming and expensive job. Consequently, it is necessary to design the DL-based models that lead to better learning from a restricted fundus image dataset. Typically, in such instance of implementing an automatic clarification for DR retinal fundus images, an additional class imbalance issue may happen. Hence, it is noteworthy to explore the class imbalance issue for enhancing the DL-based models on considering a particular class learning bias.

\section{CONCLUSIONS}

This research work has provided a detail description on various methods used for DR detection and classification with their benefits and limitations. This survey has explained the details on the various contributed methods. From the conclusion, it can be seemed that

Published By:

Blue Eyes Intelligence Engineering \& Sciences Publication (C) Copyright: All rights reserved. 
- This survey has made an review over 30 research papers related to DR detection and classification methods.

- At first, the algorithm that used in the contributed papers was categorized based on some existing models.

- Further, the analysis was extended over the performance parameters that used and their attained best values.

- At last, the research issues related to these DR detection models along with their challenges were explained to endure and rectify the future issues.

\section{REFERENCES}

1. Shaohua Wan, Yan Liang, Yin Zhang," Deep convolutional neural networks for diabetic retinopathy detection by image classification", Computers \& Electrical Engineering, vol.72, pp.274-282, November 2018

2. Javeria Amin, Muhammad Sharif, Mussarat Yasmin, Hussam Ali, Steven Lawrence Fernandes," A method for the detection and classification of diabetic retinopathy using structural predictors of bright lesions", Journal of Computational Science, vol.19, pp.153164, March 2017

3. M. Usman Akram, Shehzad Khalid, Shoab A. Khan," Identification and classification of microaneurysms for early detection of diabetic retinopathy", Pattern Recognition, vol.46, no.1, pp.107-116, January 2013

4. M. Usman Akram, Shehzad Khalid, Anam Tariq, Shoab A. Khan, Farooque Azam," Detection and classification of retinal lesions for grading of diabetic retinopathy", Computers in Biology and Medicine, vol.45, pp.161-171, 1 February 2014

5. R. A. Welikala, M. M. Fraz, J. Dehmeshki, A. Hoppe, S. A. Barman," Genetic algorithm based feature selection combined with dual classification for the automated detection of proliferative diabetic retinopathy", Computerized Medical Imaging and Graphics, vol.43, pp.64-77, July 2015

6. Tahira Nazir, Aun Irtaza, Zain Shabbir, Ali Javed, Muhammad Tariq Mahmood," Diabetic retinopathy detection through novel tetragonal local octa patterns and extreme learning machines", Artificial Intelligence in Medicine, vol.99, August 2019

7. T. Shanthi, R. S. Sabeenian," Modified Alexnet architecture for classification of diabetic retinopathy images", Computers \& Electrical Engineering, vol.76, pp.56-64, June 2019

8. Ramon Pires, Sandra Avila, Jacques Wainer, Eduardo Valle, Anderson Rocha," A data-driven approach to referable diabetic retinopathy detection", Artificial Intelligence in Medicine, vol.96, pp.93-106, May 2019

9. Turab SELÇUK, Ahmet ALKAN," Detection of microaneurysms using ant colony algorithm in the early diagnosis of diabetic retinopathy", Medical Hypotheses, vol.129, August 2019

10. Zeynab Mohammadpoory, Mahda Nasrolahzadeh, Naghmeh Mahmoodian, Javad Haddadnia," Automatic identification of diabetic retinopathy stages by using fundus images and visibility graph method", Measurement, vol.140, pp.133-141, July 2019

11. L. Seoud, T. Hurtut, J. Chelbi, F. Cheriet and J. M. P. Langlois, "Red Lesion Detection Using Dynamic Shape Features for Diabetic Retinopathy Screening," in IEEE Transactions on Medical Imaging, vol. 35, no. 4, pp. 1116-1126, April 2016.

12. B. Zhang, B. V. K. Vijaya Kumar and D. Zhang, "Detecting Diabetes Mellitus and Nonproliferative Diabetic Retinopathy Using Tongue Color, Texture, and Geometry Features," in IEEE Transactions on Biomedical Engineering, vol. 61, no. 2, pp. 491-501, Feb. 2014.

13. X. Zeng, H. Chen, Y. Luo and W. Ye, "Automated Diabetic Retinopathy Detection Based on Binocular Siamese-Like Convolutional Neural Network," in IEEE Access, vol. 7, pp. 3074430753, 2019.

14. R. Pires, H. F. Jelinek, J. Wainer, S. Goldenstein, E. Valle and A. Rocha, "Assessing the Need for Referral in Automatic Diabetic
Retinopathy Detection," in IEEE Transactions on Biomedical Engineering, vol. 60, no. 12, pp. 3391-3398, Dec. 2013.

15. B. Antal and A. Hajdu, "An Ensemble-Based System for Microaneurysm Detection and Diabetic Retinopathy Grading," in IEEE Transactions on Biomedical Engineering, vol. 59, no. 6, pp. 1720-1726, June 2012.

16. P. Costa, A. Galdran, A. Smailagic and A. Campilho, "A WeaklySupervised Framework for Interpretable Diabetic Retinopathy Detection on Retinal Images," in IEEE Access, vol. 6, pp. 1874718758, 2018.

17. L. Zhou, Y. Zhao, J. Yang, Q. Yu and X. Xu, "Deep multiple instance learning for automatic detection of diabetic retinopathy in retinal images," in IET Image Processing, vol. 12, no. 4, pp. 563-571, 42018.

18. D. Jude Hemanth, Omer Deperlioglu, Utku Kose," An enhanced diabetic retinopathy detection and classification approach using deep convolutional neural network", Neural Computing and Applications, pp 1-15, 01 January 2019

19. Sarni Suhaila Rahim, Vasile Palade, James Shuttleworth, Chrisina Jayne," Automatic screening and classification of diabetic retinopathy and maculopathy using fuzzy image processing", Brain Informatics, vol.3, no.4, pp 249-267, December 2016

20. Sarni Suhaila Rahim, Chrisina Jayne, Vasile Palade, James Shuttleworth," Automatic detection of microaneurysms in colour fundus images for diabetic retinopathy screening", Neural Computing and Applications, vol.27, no.5, pp 1149-1164, July 2016

21. Usman M. Akram, Shoab A. Khan," Automated Detection of Dark and Bright Lesions in Retinal Images for Early Detection of Diabetic Retinopathy", Journal of Medical Systems, Volume 36, Issue 5, pp 3151-3162, October 2012

22. Sabyasachi ChakrabortyGopal Chandra JanaDivya KumariAleena Swetapadma," An improved method using supervised learning technique for diabetic retinopathy detection", International Journal of Information Technology, pp 1-5, 28 May 2019

23. Karthikeyan Ganesan, Roshan Joy Martis, U. Rajendra Acharya, Chua Kuang Chua, Lim Choo Min, E. Y. K. Ng, Augustinus Laude," Computer-aided diabetic retinopathy detection using trace transforms on digital fundus images", Medical \& Biological Engineering \& Computing, vol.52, no.8, pp 663-672, August 2014

24. Hao Pang, Chang Luo, Cong Wang," Improvement of the Application of Diabetic Retinopathy Detection Model", Wireless Personal Communications, Volume 103, Issue 1, pp 611-624, November 2018

25. Hossein Ghayoumi Zadeh, Hamidreza Jamshidi, Ali Fayazi, Mohammad Hossein Gholizadeh, Cyrus Ahmadi Toussi, Mostafa Danaeian," A new model for retinal lesion detection of diabetic retinopathy using hierarchical self-organizing maps", Iran Journal of Computer Science, pp 1-9, 20 June 2019

26. C. Raja, L. Balaji," An Automatic Detection of Blood Vessel in Retinal Images Using Convolution Neural Network for Diabetic Retinopathy Detection", Pattern Recognition and Image Analysis, vol.29, no.3, pp 533-545, July 2019

27. R. Karthikeyan, P. Alli," Feature Selection and Parameters Optimization of Support Vector Machines Based on Hybrid Glowworm Swarm Optimization for Classification of Diabetic Retinopathy", Journal of Medical Systems, October 2018

28. Karkuzhali S, Manimegalai D," Distinguising Proof of Diabetic Retinopathy Detection by Hybrid Approaches in Two Dimensional Retinal Fundus Images", Journal of Medical Systems, June 2019

29. Somasundaram S K, Alli P," A Machine Learning Ensemble Classifier for Early Prediction of Diabetic Retinopathy", Journal of Medical Systems, December 2017

30. Yo-Ping Huang, Haobijam Basanta, Tzu-Hao Wang, Hung-Chou Kuo, Wei-Chi Wu," A Fuzzy Approach to Determining Critical Factors of Diabetic Retinopathy and Enhancing Data Classification Accuracy", International Journal of Fuzzy Systems, vol.21, no.6, pp 1844-1857, September 2019 